\title{
Evidence of Neuroplasticity and Neuroimaging Techniques following Anterior Cruciate Ligament Injury
}

\author{
Yong Woo An \\ Department of Kinesiology and Dance, New Mexico State University, Las Cruces, NM, USA
}

PURPOSE: Recent evidence has linked neural changes in the brain, known as neuroplasticity, to anterior cruciate injury, possibly explaining why some patients suffer repetitive functional instability. Therefore, the purpose of this article was to review the manifestation of central nervous system reorganization following anterior cruciate ligament injury and establish further the application of neuroimaging assessment tools in the evaluation of neuroplasticity after ligamentous injury.

METHODS: Previous research articles emphasizing neuromuscular deficits, functional joint instability, and anterior cruciate ligament injury and neuroimaging techniques searched from PubMed databases were reviewed. Additionally, more relevant research articles through a cited reference search from the original research articles were reviewed.

RESULTS: Diminished neuromuscular control such as proprioception, muscle activation, and joint stiffness regulation appears to be indirect evidence of neural adaptations that may lead to persistent functional joint instability and future pathological complications. Results of direct measurement of the brain's function using a variety of neuroimaging tools supports altered cortical activation in the brain corresponding to diminished knee function.

CONCLUSIONS: Knee functional deficits after an anterior cruciate ligament injury may be associated with neural adaptations in the brain. Neuroimaging techniques that allow for examination of neural activity in the central nervous system help to better understand the neurophysiological mechanisms underlying the link between the brain's function and functional joint instability, to develop more effective rehabilitation programs, and to improve patient outcomes.

Key words: Anterior cruciate ligament injury, Functional joint instability, Neuroplasticity, Neuroimaging technique

\section{INTRODUCTION}

After a tear of the anterior cruciate ligament (ACL), persistent knee functional deficits such as experiences of repetitive joint "giving way," known as functional joint instability, have received great attention by many investigators because of the healthcare burden, low success rates of return to pre-injury levels of physical activity, and higher incidence rates of recurrent rupture [1-7]. Moreover, these diminished knee functions and loss of dynamic stability are observed not only in the injured knee, but also in the contralateral knee in which the ACL is still intact, regardless of a contact or non-contact mechanism [5,8-11]. Damage to the ACL can lead to deafferentation of mechanoreceptors in the knee $[12,13]$ and it may alter sensitivity of the neural connectivity within the central nervous system (CNS) [14-16]. This diminished sensory integration between the injured ACL and CNS may interfere with the normal neural processing associated with the uninjured knee function [17]. Therefore, identifying neurophysiological alterations that may be associated with functional joint instability after an ACL injury is critical to prevent recurrent ligamentous tears and to develop rehabilitation programs to optimize patient outcomes without persistent clinical impairments.

The neuromuscular control contributing to maintenance of functional joint stability is regulated by the integration of significant proprioceptive information and cognitive learning processing based on previous physical performance $[18,19]$. This may be an indication of continuous

Corresponding author: Yong Woo An Tel +1-575-646-1636 Fax +1-575-646-4065 E-mail anyonganmsu.edu

Received 26 Feb 2018 Revised 12 Mar 2018 Accepted 19 Apr 2018

(a) This is an Open Access article distributed under the terms of the Creative Commons Attribution Non-Commercial License (http://creativecommons.org/licenses/by-nc/4.0/) which permits unrestricted non-commercial use, distribution, and reproduction in any medium, provided the original work is properly cited. 
cortical adaptation of existing neural networks at different levels of the CNS [20,21]. Recent evidence has linked reorganization of the CNS, which is known as neuroplasticity, to decreased knee function after ACL injury. This may possibly explain why some patients that suffer repetitive functional instability have recurrent knee sprains and/or contralateral injuries, and fail to return to pre-injury levels of physical activity, despite restoration of mechanical laxity $[17,22,23]$. In addition, prolonged disruption of the CNS in conjunction with the bilateral joint deficits may also imply that the ACL injury also induces neural adaptations in the CNS associated with function of the contralateral knee [17]. A variety of neuroimaging techniques evaluating neurophysiological events in the brain suggest that a tear of the ACL may alter the normal cascade of neural processing responsible for both sensory perception and motor output in the CNS [24-28]. Therefore, the purpose of this review article is to provide a comprehensive and succinct review of the evidence for neuroplastic changes in the CNS after ACL injury and an overview of neuroimaging techniques that can be utilized in examining neurophysiological responses in the brain in ACL patients.

\section{NEUROPHYIOLOGICAL DEFICTS AFTER ACL INJURY}

Following an ACL injury, dissimilar neuromuscular control responses such as altered proprioception and muscle stiffness regulation strategies have been observed among ACL patients [29-31]. Diminished proprioception and muscle coordination failure during physical activity may be an indication of indirect evidence of neuroplastic changes in the CNS after an ACL injury. Beard et al. [32] reported the timing of reflex hamstring contraction latency (RHCL) as an indirect measure of knee proprioception in ACL deficient (ACLD) patients who had conservative rehabilitation program without a surgical procedure. It was found that ACLD individuals showed $46.1 \mathrm{~ms}$ slower RHCL in the injured limb than the uninjured limb, when compared to the interlimb difference in healthy controls (4.2 ms). These patients also reported a higher frequency of "giving-way" episodes, regardless of joint laxity. Such ligament-muscle reflex latency changes observed in these patients may be indication of alteration in afferent integration processes from the muscle spindle system, which may change the muscle inhibitory or excitatory strategies implicated in the neuromuscular control $[33,34]$.

Furthermore, a number of studies have also reported conflicting results on joint position sense awareness in ACL reconstruction (ACLR) patients who had a surgical repair and were expected to have restoration of mechanical stability, with the re-innervation of ligament mechanoreceptors providing sufficient knee joint proprioceptive information to the CNS. Angoules et al. [35] and Mir et al. [36] demonstrated that ACLR patients had a restored ability to detect passive joint position during flexion and extension of the reconstructed knee. However, diminished sensation of active joint position reproduction and detection of passive knee motion were also observed in the ACLR patients when comparing performance to healthy controls [37]. These proprioceptive deficits are also observed in the opposite intact limb in ACL patients, regardless of surgical procedure [8]. Some ACL noncopers, who failed to return to pre-injury levels of physical function with experiences of functional joint instability, have developed bilateral knee dysfunction and suffered a secondary rupture of their ACL, not only in the ipsilateral knee but also the contralateral side [5,8-11]. Arockiaraj et al. [8] reported diminished balancing ability in both the injured and uninjured knees and increased errors of the threshold detection of passive movement (TDPM). This may indicate that the occurrence of ACL rupture would result in permanent modification of cortical networks (CNS reorganization) implicated in proprioceptive feedback mechanisms, thus interrupting the dynamic restraint system [17,38].

Following an ACL injury, ACLD patients have demonstrated quadriceps inhibition following knee perturbations [39,40]. Swanik et al. [41] showed that an ACLD group displayed attenuated quadriceps, and exhibited greater hamstrings reactive activation in response to joint loading during high velocity movement tasks (landing and running), when compared to healthy controls. Results also showed that this neuromuscular control deficit occurred in both the deficient and healthy knees. As the hamstrings and quadriceps muscles reciprocally inhibit each other [42], this information may indicate that the CNS reorganizes the neuromuscular control system to sustain functional stability by recruiting more knee flexors activity and decreasing extensor responses, thus minimizing excessive anterior shear forces and joint translation. This hamstrings exhibition is also observed for preparatory muscle activation processes [41].

Swanik et al. [19] also reported that ACLD patients had enhanced preparatory activity of the hamstring muscles, which acts as a restraint to anterior displacement of the ACL during landing tasks. Although each patient had varied amounts of anterior joint laxity, reactive muscle patterns and functional performance did not differ between ACLD patients and healthy controls. Increased hamstring preparatory activity was also 
observed in ACLD individuals with better knee function during dynamic deceleration of the knee muscles when completing the landing motion of a single-leg hop [43]. Conversely, other studies have reported that ACL patients with long-term disability show no significant differences in the preparatory muscle activation patterns (i.e. quadriceps inhibition) between injured and non-injured knees or compared with healthy controls during a landing task $[44,45]$. These findings imply that ACL copers, who successfully return to normal physical functions after an ACL injury, compensate for neuromechanical decoupling between the knee and CNS, regardless of joint laxity. Therefore, ACL copers can protect knee joints bilaterally by optimizing muscle contraction strategies to maintain functional joint stability during a complex physical activity known to stress the ACL, whereas ACL noncopers do not $[2,18,19,46]$.

Moreover, several subsequent ACL injury studies have reported that once ACL patients are cleared to return to normal activity without functional limitations, the incidence rate of a second ACL rupture after a reconstruction increases from $6 \%$ within 2 years [47], to $12 \%$ within 5 years [48], and almost 30\% within 10 years [49]. It has also been reported that the risk of a subsequent ACL rupture to the opposite limb is greater than the ipsilateral limb after the reconstruction, particularly in younger patients or with intensive activities [11,48,50]. Evidence of the development of proprioceptive and neuromuscular control deficits leading to secondary ACL sprains to the contralateral side may be indicative of persistent neural maladaptation in the cerebral cortex, which can diminish neuromuscular control system over time [26,37,51,52]. However, most of these findings are speculative, based on either indirect measures of the CNS's responses during proprioceptive tasks or clinical outcomes from prospective cohort studies. Therefore, direct observation of cortical activity in the brain should offer better insight into the manifestation of neuroplasticity in ACL patients after an ACL injury in order to precisely explore the neural origin of these neuromuscular control deficits.

\section{EVALUATION OF NEUROPLASTICITY IN ACL PATIENTS}

During the past several decades, the evolution of versatile functional neuroimaging techniques has allowed for the non-invasive exploration of cortical activation after injury [53]. Techniques that are most often used for in-vivo human brain studies can be classified into two types according to their methodological approach. The first type of functional neuroimaging technique measures neuronal metabolic changes in the cortical and/or subcortical regions, including positron emission tomography (PET), function magnetic resonance imaging (fMRI) and near infrared spectroscopy (NIRS) techniques [53-56]. When specific areas in the brain are activated in response to sensory inputs, neurons in those areas require greater supplies of glucose and oxygen to be delivered through the cerebral circulatory system to meet the neurons' increased energy demands. Therefore, an indirect measure of metabolic changes in those areas reflects the level of neural excitability or inhibition, by measuring hemodynamic responses or cerebral blood flow (CBF). Although PET and fMRI techniques can examine neuronal events both in the cerebral cortex and subcortical region of the brain, the NIRS technique is able to measure only the superficial CBF of the brain [53]. Moreover, due to the requirement of injecting a radionuclide for tracking, the PET technique carries slightly more risk relative to fMRI and NIRS. For this reason, an fMRI technique has been used to examine neural adaptations following an ACL injury.

Kapreli et al. [24] found changes in cortical activation patterns in the CNS during a simple knee flexion/extension task observed by using fMRI technique among ACLD patients with prolonged functional disability. In comparison with healthy controls, the ACLD individuals revealed reduced cortical activation in several cerebral and subcortical areas, including somatosensory and premotor cortices and thalamus, which are regions associated with regulation of sensory perception and motor output. On the contrary, these patients showed higher activation in some other cortical regions including the visual and primary motor cortices, which are proposed to be critical for preparatory feed-forward mechanism $[57,58]$. The author suggested that the increased neural demand of visual perception could compensate for diminished proprioception in ACLD patients by enhancing recognition of significant visual cues for early planning of movement [24]. Grooms et al. [59] also demonstrated altered cortical activation in ACLR patients with fMRI during active knee flexion/extension motor task. The results supported that neural activation patterns in the brain may be altered as a result of ACL injury [24], but showed more cortical activation in the sensorimotor cortices and cerebellum when compared to healthy controls. This increased somatosensory cortex activation may be an alternative protective compensatory mechanism for increased neural demands as a result of decreased proprioception after ACL injury [28]. This fMRI technique provides better spatial resolution, which is the accuracy in the location and dimension of brain activity $[53,60]$. As chronic functional deficits after an ACL injury may manifest permanent neural maladaptation in the 
brain [61], fMRI techniques may help to identify the origin of the neuroplasticity. However, it has limitations to adequately offer observation of the critical neural mechanisms underlying neuromuscular control during unanticipated events in physical activity that lead to functional instability episodes $[53,62]$. Observing metabolic changes in cortical neurons requires a few seconds of temporal resolution, which refers to the accuracy in real time of the cascade of cortical activation within and between areas in the brain $[53,62]$. ACL injuries can occur in less than $70 \mathrm{~ms}$ and the neuromuscular control system can regulate muscle stiffness strategies in less than $50 \mathrm{~ms}[63,64]$. Therefore, the fMRI's temporal resolution is too slow for accurately measuring cortical events within the injury timeline of interest [53].

The second group of measurement techniques for brain activity is growing in popularity, including transcranial magnetic stimulation (TMS), electroencephalography (EEG) and magnetoencephalography (MEG) techniques $[53,65]$. These techniques provide excellent temporal resolution in milliseconds, and allow for the measurement of simultaneous cortical responses at the brain's raw "speed of thought" $[53,62]$. When cortical neurons are activated, postsynaptic potentials produce small, fluctuating electrical ionic currents, as well as small magnetic field oscillations. The strength of these electrical currents implies the level of cortical activation. The TMS technique, more precisely, generates artificial electrical currents, which modifies the neurons' output excitability, by delivering a magnetic pulse into specific areas of the cerebral cortex [66]. Kuenze et al. [52] used the TMS technique to examine motor-evoked potentials (MEPs) of the primary motor cortex during isometric knee extension contraction at $5 \%$ of maximum voluntary isometric contractions (MVICs). In comparison with uninjured knees, reconstructed knees of ACLI patients revealed greater MEPs, but they did not differ from the bilateral knees of healthy controls. It is known that a greater MEP indicates less cortical excitability and facilitation of muscle contractions [67]. Given this fact, the results of this study may indicate that an ACL injury caused long-term muscle weakness in the reconstructed knee because the motor cortex was providing insufficient stimulus during physical activity $[68,69]$. Contralateral cortical excitability patterns were also observed by Heroux and Tremblay [66] and Pietrosimone et al. [69]. These studies found that ACLR patients had increased excitability in their reconstructed knee compared to their uninvolved knee. From these combined results, the asymmetric corticospinal excitability over the primary motor cortex may imply that changes in neurophysiological networks at the cortex level would interfere with both the reconstructed and contralater- al limbs' dynamic restraint needed for the maintenance of functional joint stability following ACL injury [21]. Therefore, this TMS technique is an excellent neuroimaging tool to identify the relationship between detection of proprioceptive inputs at the somatosensory cortex and an efferent neuronal excitability at the corticomotor level, reflecting reactive muscle activity through the proprioceptive feedback mechanism [66]. However, it may not be a proper technique to investigate how the brain detects external and internal stimuli and controls preparation of bodily movement in advance, which are necessary to maintain functional joint stability.

Substantial advantages of EEG and MEG over other noninvasive functional neuroimaging of the brain, such as fMRI and PET, are the level of temporal resolution in the order of milliseconds, as well as direct recording of the cascade of neuronal electrical currents of the entire cerebral cortex $[53,62]$. Furthermore, EEG is portable, relatively cheap, and does not require a large space for the test setting, in contrast to stationary PET, fMRI and MEG, which are large and can cost millions [53]. Thus, high temporal resolution and observation of concurrent neural activation across cortical areas with EEG may provide the opportunity for the examination of highly transient brain source activities implicated in perception, motor planning, and execution of motor control after an ACL injury $[53,70]$.

EEG recordings have shown a variety of frequency bands such as Delta (<4 Hz), Theta (4-8 Hz), Alpha-1 (8-10 Hz), Alpha-2 (10-12 Hz), Beta (1631), Gamma (>32), and Mu (8-12) in the human's brain [71-73]. With regards to neuromuscular control, the fast Alpha-2 frequency band in the parietal brain regions is concerned with sensorimotor neurons' excitation and inhibition during a motor task, while the Theta frequency band in the frontal brain area is associated with task-related cognitive processing, as well as emotional regulation [74-76]. Baumeister et al. [25,26] demonstrated in EEG studies that ACLR patients had dissimilar cortical activation in the frontal and parietal cortices during force and/or joint position reproduction tasks, when compared to healthy controls. When ACLR patients performed force or joint reproduction tasks, they had increased frontal theta frequency power, reflecting augmented cortical activation in the anterior cingulate cortex (ACC) responsible for cognitive motor processing [77], thus supporting the findings reported by Kapreli et al. [24]. Furthermore, during the joint reproduction task, ACLR patients revealed significant reduction in Alpha-2 parietal (P3,P4) frequency powers, indicating higher cortical activation in the parietal sensorimotor cortex in order to better perceive, process and integrate decreased 
proprioceptive inputs after ACL injury [28]. Moreover, cortical connectivity between the frontal and parietal cortex is known as a neural network for working memory, which relates to short-term memory abilities of monitoring, maintaining and modulating information for goal-directed behaviors [78]. Enhanced neural activity occurring simultaneously in these cortices reflects heightened neurocognitive processing [26,79]. As a result, these findings may support the notion that the modified CNS after injury, must recruit more neural resources in the planning of movement to compensate for diminished sensory feedback information through neurocognitive processing $[19,80]$. However, the CNS reorganization, as a result of an ACL rupture, may not provide enough neural resources in response to sudden knee perturbation, as brain regions for task-related cognitive processing could be compromised after the ACL rupture, leading to interrupted neuromuscular control. For this reason, an individual's executive function capabilities may be linked to ACL injury proneness, and may have a substantial role in restoration and maintenance of functional joint stability following an ACL injury $[81,82]$.

\section{CONCLUSION}

Regardless of injury mechanisms (contact or non-contact) or treatment of option (surgical or conservative), an ACL injury could lead to failure in muscle coordination and development of pathological complications such as a recurrent rupture to either ipsilateral or contralateral and early onset of knee osteoarthritis. Changes of neural activity in the brain have been thought to be the primary reason for functional limitations after the ACL injury. Diminished proprioception, such as greater errors in joint position and force reproduction, not only in the injured limb but also in the healthy knee and the altered muscle contraction strategies; i.e. decreased joint stiffness to a sudden perturbation or excessive or less muscle activation, may be indirect evidence of insufficient neural adaption in the CNS. Moreover, recent knee studies that utilize versatile functional neuroimaging techniques allow direct examination of cortical activation in the brain in ACL patients corresponding to knee function tasks, and the results of these studies have demonstrated that altered brain function in ACL patients is highly associated with diminished knee proprioception and motor control. To date, the results of the neuroimaging studies are not yet conclusive in terms of identification of the interrelationship between knee function and neural adaption in the CNS underlying functional joint instability. Moreover, due to the limitation of the nature of neuroimaging study setting, it is important that in- vestigators understand the advantage and disadvantage of each neuroimaging techniques and choose an appropriate technique according to the purpose of the study. Future study combining advantage of each neuroimaging technique may provide further insight into the structural and functional neural adaptation in the brain after an ACL injury and it will advance our knowledge on the role of the brain associated with functional joint instability and help to develop rehabilitation programs and improve patient outcomes [83].

\section{REFERENCES}

1. Gobbi A, Bathan L, Boldrini L. Primary repair combined with bone marrow stimulation in acute anterior cruciate ligament lesions: results in a group of athletes. Am J Sports Med. 2009;37(3):571-8.

2. Kaplan Y. Identifying individuals with an anterior cruciate ligamentdeficient knee as copers and noncopers: a narrative literature review. J Orthop Sports Phys Ther. 2011;41(10):758-66.

3. Dhillon MS, Bali K, Prabhakar S. Proprioception in anterior cruciate ligament deficient knees and its relevance in anterior cruciate ligament reconstruction. Indian J Orthop. 2011;45(4):294-300.

4. Fitzgerald GK, Axe MJ, Snyder-Mackler L. A decision-making scheme for returning patients to high-level activity with nonoperative treatment after anterior cruciate ligament rupture. Knee Surg Sports Traumatol Arthrosc. 2000;8(2):76-82.

5. Grindem H, Eitzen I, Moksnes H, Snyder-Mackler L, Risberg MA. A pair-matched comparison of return to pivoting sports at 1 year in anterior cruciate ligament-injured patients after a nonoperative versus an operative treatment course. Am J Sports Med. 2012;40(11):2509-16.

6. Herrington L, Fowler E. A systematic literature review to investigate if we identify those patients who can cope with anterior cruciate ligament deficiency. Knee. 2006;13(4):260-5.

7. Lee DYH, Karim SA, Chang HC. Return to sports after anterior cruciate ligament reconstruction - a review of patients with minimum 5-year follow-up. Ann Acad Med Singapore. 2008;37(4):273-8.

8. Arockiaraj J, Korula RJ, Oommen AT, Devasahayam S, Wankhar S, et al. Proprioceptive changes in the contralateral knee joint following anterior cruciate injury. Bone Joint J. 2013;95(2):188-91.

9. Paterno MV, Rauh MJ, Schmitt LC, Ford KR, Hewett TE. Incidence of second ACL injuries 2 years after primary ACL reconstruction and return to sport. Am J Sports Med. 2014;42(7):1567-73.

10. Paterno MV, Rauh MJ, Schmitt LC, Ford KR, Hewett TE. Incidence of 
contralateral and ipsilateral anterior cruciate ligament (ACL) injury after primary ACL reconstruction and return to sport. Clin J Sport Med. 2012;22(2):116-21.

11. Webster KE, Feller JA, Leigh WB, Richmond AK. Younger patients are at increased risk for graft rupture and contralateral injury after anterior cruciate ligament reconstruction. Am J Sports Med. 2014;42(3):641-7.

12. Denti M, Monteleone M, Berardi A, Panni AS. Anterior cruciate ligament mechanoreceptors. Histologic studies on lesions and reconstruction. Clin Orthop Relat Res. 1994;(308):29-32.

13. Shimizu T, Takahashi T, Wada Y, Tanaka M, Morisawa Y, et al. Regeneration process of mechanoreceptors in the reconstructed anterior cruciate ligament. Arch Orthop Trauma Surg. 1999;119(7-8):405-9.

14. Johansson H, Sjölander P, Sojka P. Receptors in the knee joint ligaments and their role in the biomechanics of the joint. Crit Rev Biomed Eng. 1991;18(5):341-68.

15. Johansson H, Sjölander P, Sojka P. Activity in receptor afferents from the anterior cruciate ligament evokes reflex effects on fusimotor neurones. Neurosci Res. 1990;8(1):54-9.

16. An YW. Neurophysiological mechanisms underlying functional knee instability following an anterior cruciate ligament injury. Exer Sci. 2018;27(2):109-17.

17. Kapreli E, Athanasopoulos S. The anterior cruciate ligament deficiency as a model of brain plasticity. Med Hypotheses. 2006;67(3):645-50.

18. Riemann BL, Lephart SM. The sensorimotor system, part II: the role of proprioception in motor control and functional joint stability. J Athl Train. 2002;37(1):80-4.

19. Swanik CB, Lephart SM, Swanik KA, Stone DA, Fu FH. Neuromuscular dynamic restraint in women with anterior cruciate ligament injuries. Clin Orthop Relat Res. 2004;(425):189-99.

20. Cramer SC, Sur M, Dobkin BH, O’Brien C, Sanger TD, et al. Harnessing neuroplasticity for clinical applications. Brain. 2011;134(6):1591609.

21. Dayan E, Cohen LG. Neuroplasticity subserving motor skill learning. Neuron. 2011;72:443-54.

22. Courtney Ca, Rine RM. Central somatosensory changes associated with improved dynamic balance in subjects with anterior cruciate ligament deficiency. Gait Posture. 2006;24(2):190-5.

23. Courtney C, Rine RM, Kroll P. Central somatosensory changes and altered muscle synergies in subjects with anterior cruciate ligament deficiency. Gait Posture. 2005;22(1):69-74.

24. Kapreli E, Athanasopoulos S, Gliatis J, Papathanasiou M, Peeters R, et al. Anterior cruciate ligament deficiency causes brain plasticity: a functional MRI study. The Am J Sports Med. 2009;37(12):2419-26.

25. Baumeister J, Reinecke K, Schubert M, Weiss M. Altered electrocortical brain activity after ACL reconstruction during force control. J Orthop Res. 2011;29(9):1383-9.

26. Baumeister J, Reinecke K, Weiss M. Changed cortical activity after anterior cruciate ligament reconstruction in a joint position paradigm: an EEG study. Scand J Med Sci Sports. 2008;18(4):473-84.

27. Grooms D, Appelbaum G, Onate J. Neuroplasticity following anterior cruciate ligament injury: a framework for visual-motor training approaches in rehabilitation. J Orthop Sports Phys Ther. 2015;45(5):38193.

28. Grooms DR, Page S, Onate JA. Brain activation for knee movement measured days before second anterior cruciate ligament Injury: neuroimaging in musculoskeletal medicine. J Athl Train. 2015;50(10):1005-10.

29. Ageberg E, Björkman A, Rosén B, Roos EM. Principles of brain plasticity in improving sensorimotor function of the knee and leg in patients with anterior cruciate ligament injury: a double-blind randomized exploratory trial. BMC Musculoskelet Disord. 2012;13(1):68.

30. Ageberg E, Thomeé R, Neeter C, Silbernagel KG, Roos EM. Muscle strength and functional performance in patients with anterior cruciate ligament injury treated with training and surgical reconstruction or training only: a two to five-year followup. Arthritis Rheum. 2008; 59(12):1773-9.

31. Gobbi A, Francisco R. Factors affecting return to sports after anterior cruciate ligament reconstruction with patellar tendon and hamstring graft: a prospective clinical investigation. Knee Surg Sports Traumatol Arthrosc. 2006;14(10):1021-8.

32. Beard DJ, Kyberd PJ, Fergusson CM, Dodd CA. Proprioception after rupture of the anterior cruciate ligament. An objective indication of the need for surgery?. J Bone Joint Surg Br. 1993;75(2):311-5.

33. Friemert B, Faist M, Spengler C, Gerngross H, Claes L, et al. Intraoperative direct mechanical stimulation of the anterior cruciate ligament elicits short- and medium-latency hamstring reflexes. J Neurophysiol. 2005;94(6):3996-4001.

34. Johansson H, Sjölander P, Sojka P. A sensory role for the cruciate ligaments. Clin Orthop Relat Res. 1991;(268):161-78.

35. Angoules AG, Mavrogenis AF, Dimitriou R, Karzis K, Drakoulakis E, et al. Knee proprioception following ACL reconstruction; a prospective trial comparing hamstrings with bone-patellar tendon-bone autograft. Knee. 2011;18(2):76-82. 
36. Mir SM, Hadian MR, Talebian S, Nasseri N. Functional assessment of knee joint position sense following anterior cruciate ligament reconstruction. Br J Sports Med. 2008;42(4):300-3.

37. Bonfim TR, Jansen Paccola CA, Barela JA. Proprioceptive and behavior impairments in individuals with anterior cruciate ligament reconstructed knees. Arch Phys Med Rehab. 2003;84(8):1217-23.

38. Ward S, Pearce AJ, Pietrosimone B, Bennell K, Clark R, et al. Neuromuscular deficits after peripheral joint injury: a neurophysiological hypothesis. Muscle Nerve. 2015;51(3):327-32.

39. Di Fabio RP, Graf B, Badke MB, Breunig A, Jensen K. Effect of knee joint laxity on long-loop postural reflexes: evidence for a human capsular-hamstring reflex. Exp Brain Res. 1992;90(1):189-200.

40. Rice DA, McNair PJ. Quadriceps arthrogenic muscle inhibition: neural mechanisms and treatment perspectives. Semin Arthritis Rheum. 2010;40(3):250-66.

41. Swanik CB, Lephart SM, Giraldo JL, Demont RG, Fu FH. Reactive muscle firing of anterior cruciate ligament-injured females during functional activities. J Athl Train. 1999;34(2):121-9.

42. Begalle RL, DiStefano LJ, Blackburn T, Padua Da. Quadriceps and hamstrings coactivation during common therapeutic exercises. J Athl Train. 2012;47(4):396-405.

43. Bryant AL, Newton RU, Steele J. Successful feed-forward strategies following ACL injury and reconstruction. J Electromyogr Kinesiol. 2009; 19(5):988-97.

44. Gauffin H, Tropp H. Altered movement and muscular-activation patterns during the one-legged jump in patients with an old anterior cruciate ligament rupture. Am J Sports Med. 1992;20(2):182-92.

45. McNair PJ, Marshall RN. Landing characteristics in subjects with normal and anterior cruciate ligament deficient knee joints. Arch Phys Med Rehab. 1994;75(5):584-9.

46. DeAngelis AI, Needle AR, Kaminski TW, Royer TR, Knight CA, et al. An acoustic startle alters knee joint stiffness and neuromuscular control. Scand J Med Sci Sports. 2015;25(4):509-16.

47. Wright RW, Dunn WR, Amendola A, Andrish JT, Bergfeld J, et al. Risk of tearing the intact anterior cruciate ligament in the contralateral knee and rupturing the anterior cruciate ligament graft during the first 2 years after anterior cruciate ligament reconstruction: a prospective MOON cohort study. Am J Sports Med. 2007;35(7):1131-4.

48. Salmon L, Russell V, Musgrove T, Pinczewski L, Refshauge K. Incidence and risk factors for graft rupture and contralateral rupture after anterior cruciate ligament reconstruction. Arthroscopy. 2005;21(8):948-57.
49. Pinczewski LA, Lyman J, Salmon LJ, Russell VJ, Roe J, et al. A 10-year comparison of anterior cruciate ligament reconstructions with hamstring tendon and patellar tendon autograft: a controlled, prospective trial. Am J Sports Med. 2007;35(4):564-74

50. Wright RW, Magnussen RA, Dunn WR, Spindler KP. Ipsilateral graft and contralateral ACL rupture at five years or more following ACL reconstruction: a systematic review. J Bone Joint Surg Am. 2011;93(12) 1159-65.

51. Hiemstra LA, Webber S, MacDonald PB, Kriellaars DJ. Contralateral limb strength deficits after anterior cruciate ligament reconstruction using a hamstring tendon graft. Clin Biomech. 2007;22(5):543-50.

52. Kuenze CM, Hertel J, Weltman A, Diduch D, Saliba Sa, et al. Persistent neuromuscular and corticomotor quadriceps asymmetry after anterior cruciate ligament reconstruction. J Athl Train. 2015;50(3):303-12.

53. Crosson B, Ford A, McGregor KM, Meinzer M, Cheshkov S, et al Functional imaging and related techniques: an introduction for rehabilitation researchers. J Rehab Res Dev. 2010;47(2):7-34.

54. Kennedy SH, Evans KR, Krüger S, Mayberg HS, Meyer JH, et al. Changes in regional brain glucose metabolism measured with positron emission tomography after paroxetine treatment of major depression. Am J Psychiatry. 2001;158(6):899-905.

55. Rossini PM, Pauri F. Neuromagnetic integrated methods tracking human brain mechanisms of sensorimotor areas 'plastic’ reorganisation. Brain Research Reviews. 2000;33(2-3):131-54.

56. Yamada H, Sadato N, Konishi Y, Kimura K, Tanaka M, et al. A rapid brain metabolic change in infants detected by fMRI. Neuroreport. 1997;8(17):3775-8

57. Amaral DG. The functional organization of perception and movement, in principles of neural science. New York: McGraw-Hill. 2013;356-69.

58. Neuper C, Pfurtscheller G. Event-related dynamics of cortical rhythms: frequency-specific features and functional correlates. Int J Psychophysiol. 2001;43(1):41-58.

59. Grooms DR, Page SJ, Nichols-Larsen DS, Chaudhari AM, White SE, et al. Neuroplasticity associated with anterior cruciate ligament reconstruction. J Orthop Sports Phys Ther. 2017;47(3):180-9.

60. Mamata H, Mamata Y, Westin CF, Shenton ME, Kikinis R, et al. Highresolution line scan diffusion tensor MR imaging of white matter fiber tract anatomy. AJNR Am J Neuroradiol. 2002;23(1):67-75.

61. Nyland J, Gamble C, Franklin T, Caborn DNM. Permanent knee sensorimotor system changes following ACL injury and surgery. Knee Surg Sports Traumatol Arthrosc. 2017;25(5):1461-74. 
62. Johansson BB. Brain plasticity in health and disease. Keio J Med. 2004; 53(4):231-46.

63. Mrachacz-Kersting N, Sinkjaer T. Reflex and non-reflex torque responses to stretch of the human knee extensors. Exp Brain Res. 2003; 151(1):72-81.

64. Sinkjaer T, Toft E, Andreassen S, Hornemann BC. Muscle stiffness in human ankle dorsiflexors: intrinsic and reflex components. J Neurophysiol. 1988;60(3):1110-21.

65. Pfurtscheller G, Lopes da Silva FH, Lopes FH. Event-related EEG/ MEG synchronization and desynchronization: basic principles. Clin Neurophysiol. 1999;110(11):1842-57.

66. Héroux ME, Tremblay F. Corticomotor excitability associated with unilateral knee dysfunction secondary to anterior cruciate ligament injury. Knee Surg Sports Traumatol Arthrosc. 2006;14(9):823-33.

67. Bonnard M, Camus M, de Graaf J, Pailhous J. Direct evidence for a binding between cognitive and motor functions in humans: a TMS study. J Cogn Neurosci. 2003;15(8):1207-16.

68. Howells BE, Ardern CL, Webster KE. Is postural control restored following anterior cruciate ligament reconstruction?. A systematic review. Knee Surg Sports Traumatol Arthrosc. 2011;19(7):1168-77.

69. Pietrosimone BG, Lepley AS, Ericksen HM, Gribble Pa, Levine J. Quadriceps strength and corticospinal excitability as predictors of disability after anterior cruciate ligament reconstruction. J Sport Rehabil. 2013;22(1):1-6.

70. Pfurtscheller G, Klimesch W. Event-related desynchronization during motor behavior and visual information processing. Electroencephalogr Clin Neurophysiol Suppl. 1991;42:58-65.

71. Balconi M, Lucchiari C. EEG correlates (event-related desynchronization) of emotional face elaboration: a temporal analysis. Neurosci Lett. 2006;392(1-2):118-23.

72. Balconi M, Pozzoli U. Arousal effect on emotional face comprehension: frequency band changes in different time intervals. Physiol Behav. 2009;97(3-4):455-62.
73. Pfurtscheller G, Brunner C, Schlögl A, Lopes da Silva FH. Mu rhythm (de)synchronization and EEG single-trial classification of different motor imagery tasks. Neuroimage. 2006;31(1):153-9.

74. Pfurtscheller G, Stancák A Jr, Neuper C. Event-related synchronization (ERS) in the alpha band - An electrophysiological correlate of cortical idling: A review. Int J Psychophysiol. 1996;24(1-2):39-46.

75. Tolegenova AA, Kustubayeva AM, Matthews G. Trait meta-mood, gender and EEG response during emotion-regulation. Personal Individual Differences. 2014;65:75-80.

76. Huh YS. Effects of short-term weight loss on stress hormone and EEG in weight athletes. Exer Sci. 2018;27(2):146-52.

77. Paus T. Primate anterior cingulate cortex: where motor control, drive and cognition interface. Nat Rev Neurosci. 2001;2(6):417-24.

78. Schweizer S, Grahn J, Hampshire A, Mobbs D, Dalgleish T. Training the emotional brain: improving affective control through emotional working memory training. J Neurosci. 2013;33(12):5301-11.

79. Mizelle JC, Forrester L, Hallett M, Wheaton La. Electroencephalographic reactivity to unimodal and bimodal visual and proprioceptive demands in sensorimotor integration. Exp Brain Res. 2010;203(4):65970.

80. Mizelle JC, Forrester L, Hallett M, Wheaton La. Theta frequency band activity and attentional mechanisms in visual and proprioceptive demand. Exp Brain Res. 2010;204(2):189-97.

81. Cappellino F, Paolucci T, Zangrando F, Iosa M, Adriani E, et al. Neurocognitive rehabilitative approach effectiveness after anterior cruciate ligament reconstruction with patellar tendon. A randomized controlled trial. Eur J Phys Rehabil Med. 2012;48(1):17-30.

82. Swanik CB, Covassin T, Stearne DJ, Schatz P. The relationship between neurocognitive function and noncontact anterior cruciate ligament injuries. Am J Sports Med. 2007;35(6):943-8.

83. Kim HT, Song YE, Kang EB, Cho JY, Kim BW, et al. The effects of combined exercise on basic physical fitness, neurotrophic factors and working memory of elementary students. Exer Sci. 2015;24(3):243-51. 